\title{
Mikrobiologische Aspekte der biologischen Abluftreinigung
}

K.H. Engesser

Institut für Mikrobiologie der Universität Stuttgart, Azenbergstr. 18, 7000 Stuttgart 1, FRG

\section{Zusammenfassung}

Die biologische Abluftreinigung stellt ein vielversprechendes Verfahren zur Beseitigung von Schadstoff-Emissionen dar. Ein störungsfreier Betrieb ist allerdings nur bei Einhaltung optimaler Wuchsbedingungen für die den Schadstoffabbau katalysierende, oft sehr spezialisierte Mikroorganismen-Flora möglich.

Substanzen mit xenobiotischem Charakter sind nicht ohne weiteres dem Abbau zugänglich. So ermöglicht erst die Wahl spezieller cooxidativer Prozesse den Metabolismus von hochchlorierten Ethenen. Gemische von einzeln leicht abbaubaren Substanzen können sich im Gemisch als schwerabbaubar erweisen. Erst nach Konstruktion geeigneter neuartiger Abbauwege wird ein vollständiger Abbau möglich. Die vorgestellten Beispiele belegen die Bedeutung der Grundlagenforschung im Bereich der Biodegradation für den erfolgreichen Betrieb von biologischen Abluftreinigungsanlagen unter den erschwerten Bedingungen des Abbaus von Fremdstoffen.

\section{Bedeutung der biologischen Verfahrensprinzipien}

Verfahren zur biologischen Abluftreinigung werden in jüngster Zeit zunehmend populärer [1]. Dies liegt einmal am gestiegenen Umweltbewußtsein breiter Kreise der Öfentlichkeit und damit verbunden an den zunehmend verschärften Anforderungen des Gesetzgebers an die Reinhaltung der Luft. Zum anderen hat diese Technik den Vorteil kosten-<smiles>Cc1ccccc1</smiles><smiles>CC1=CC=CC(O)C1(O)O</smiles><smiles>CCCC</smiles><smiles>Cc1cccc(O)c1O</smiles><smiles>C=CC</smiles>

Zitronensaurezyklus<smiles>[3H][Te]C</smiles><smiles>CC(=O)[OH+][CH+]C=C(O)C(=O)O</smiles><smiles>CCC</smiles><smiles>Cc1cccc(O)c1C(=O)O</smiles>

Abb. 1. Der bakterielle Abbau von Toluol 
günstig, verfahrenstechnisch einfach und genehmigungstechnisch schnell realisierbar zu sein. So dürfte es zunehmend schwerer werden, Genehmigungsverfahren für z. B. thermische Verfahren erfolgreich abzuschließen, vor allem, wenn es sich um die Beseitigung von halogenierten Kohlenwasserstoffen handelt. Da hierbei unausweichlich chlorierte Dibenzofurane und Dibenzodioxine entstehen, muß der betroffenen Öffentlichkeit die Ungefährlichkeit des Gesamtprozesses in jedem Einzelfall verstandlich gemacht werden. Ein weiterer Vorteil biologischer Reinigungsverfahren besteht in der im Idealfall vollständig verlaufenden Umwandlung von Schadstoffen in harmlose Endprodukte wie Kohlendioxid, Salze und Biomasse. Es findet also keine einfache Verlagerung ansonsten unveränderter Abfallstoffe in andere Umweltkompartimente statt. Dies ist angesichts knapper Deponiekapazitäten von größter Bedeutung.

\section{Identifizierung der Grundbedingungen biologischer Verfahren}

Mikroorganismen verfügen über ein enormes Potential zum Abbau einer extremen Vielfalt von chemischen Verbindungen unterschiedlichster Struktur [2]. Vor Einführung der sog. Xenobiotika in den Stoffkreislauf der Natur sprach man sogar von der Unfehlbarkeit der Mikroben. Durch Dissimilation von organischen und anorganischen Verbindungen gewinnen sie Energie, die sie zur Aufrechterhaltung ihres Stoffwechsels und darüberhinaus zur Vermehrung ihrer Biomasse verwenden. Voraussetzung für eine effiziente biologische Abluftreinigung ist also die biologische Abbaubarkeit der zu reinigenden Verbindungen. So können z. B. BTX-Aromaten wie Benzol, Toluol oder Xylol für viele Bakterien als Nahrungsquelle dienen [3]. Die dabei auftretenden Oxidationsprozesse sind in Abb. 1 aufgezeigt. Endprodukte sind Kohlendioxid und Zellmasse, wobei deren evtl. zu starke Zunahme zum Verstopfen von Filtermaterialien führen kann. Bei all diesen Wachstumsvorgängen ist auf die Einhaltung bestimmter Randbedingnungen zu achten: die Parameter 'relative Feuchtigkeit, " pH-Wert, Temperatur' sowie die Versorgung mit 'Nährstoffen' und 'Sauerstoff' (bei aeroben Verfahren) bzw. mit anderen Elektronenakzeptoren müssen innerhalb gewisser, für jeden Proze $B$ z. T. unterschiedlicher Grenzen geregelt werden. So sind Schimmelpilze und Hefen im Vergleich zu Bakterien unempfindlicher gegen "Wassermangel". Sie bevorzugen im allgemeinen eher saure Milieubedingungen, während Bakterien eher unter neutralen bis leicht basischen pH-Bedingungen leben. In Ausnahmefällen können Bakterien allerdings auch in stark saure oder alkalische pH-Bereiche vordringen, so Thiobacillus-Stämme bei der Oxidation von Schwefelwasserstoff zu Schwefelsäure. Bestimmte sog. alkalophile Bacillus-Stämme vermögen sich noch bei $\mathrm{pH}$-Werten von $10-11 \mathrm{zu}$ vermehren.

Hinsichtlich der Ansprüche an die optimale Temperatur für das Wachstum unterscheidet man zwischen den sog. "psychrophilen" Bakterien, deren Wachstumsoptimum unter $20^{\circ} \mathrm{C}$ liegt, während sich die "mesophilen" Stämme optimal zwischen $20^{\circ} \mathrm{C}$ und $42^{\circ} \mathrm{C}$ vermehren. Die echten "thermophilen" Bakterien haben ihr Wachstums- und Abbauleistungs-Optimum im Bereich zwischen $40^{\circ} \mathrm{C}$ und $70^{\circ} \mathrm{C}$, während einige "extrem thermophile" Bakterien bis zu $100^{\circ} \mathrm{C}$ erreichen können. So vermag Sulfolobus acidocaldarius noch bei $80^{\circ} \mathrm{C}$ Schwefel zu Schwefelsäure zu oxidieren. Hinsichtlich der Nährstoffansprüche muß ein ausgewogenes Mengenverhältnis zwischen Kohlenstoff- und Stickstoffquelle, Phosphor sowie den anderen, oft nur in Spuren benötigten Elementen bestehen. So wird eine Abluftreinigungsanlage 
für BTX-Aromaten nur dann effizient arbeiten können, wenn der inhärente Mangel an Stickstoff ausgeglichen wird. Sind hingegen organische Amine abzubauen, kann sogar ein Stickstoffüberschuß bestehen, der u.U. durch Zugabe einer Kohlenstoffquelle abgebaut werden muß.<smiles>C=CC(C)C</smiles><smiles>CCC</smiles><smiles>CCC1(C)CO1</smiles><smiles>C[Te]</smiles><smiles>CCC(C)(C)CO</smiles>

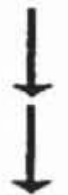<smiles>ClC=C(Cl)Cl</smiles><smiles>C[CH]C</smiles><smiles>ClC1OC1(Cl)Cl</smiles><smiles>C[14CH3]</smiles><smiles>OC(Cl)C(Cl)(Cl)Cl</smiles><smiles>C[C@@H]1CC[C]1Cl</smiles><smiles>O=CC(=O)Cl</smiles>

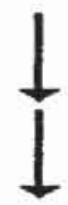

Abb. 2. Hypothetıscher Mechanismus der cometabolisch erfolgenden Dechlorierung von Trichlorethen durch Isopren-abbauende Bakterien (nach Ewers et.al., 1990) 


\section{Abbau von Problemsubstanzen}

Wie schon oben angeführt, werden sehr viele Verbindungen in Biofiltern, Biowäschern oder durch andere biologische Reinigungsverfahren mit hoher Effizienz abgebaut [4]. Dies beruht auf dem günstigen Umstand, $\mathrm{da} B$ alle diese Substanzen biologisch mehr oder weniger gut abbaubar sind und somit von den abbauenden Mikroorganismen als Nahrungs- und Energiequelle genutzt werden können.

Anders verhält es sich mit Stoffen oder Stoffgemischen, die biologisch schwer abbaubar sind. Sie sind häufig in Ablüften aus industriellen Produktionsanlagen enthalten und werden erfahrungsgemä $B$ in biologischen Reinigungsanlagen nicht oder sehr unvollständig eliminiert. Die Gründe hierfür sind ganz verschiedener Art.

\subsection{Cooxidation}

So können Stoffe zwar prinzipiell abbaubar sein, doch liefert dieser Proze $B$ keine oder nur sehr wenig Energie. Aus diesem Grunde kann sich keine aktive Bakterienflora entwickeln. Trichlorethylen oder Tetrachlorethylen z. B. (Abb. 2) kann zwar durch Methan oder Isopren abbauende Mikroorganismen verstoffwechselt werden, doch ist bei Verabreichung des chlorierten Kohlenwasserstoffes als alleinige Kohlenstoff- und Energiequelle keine Zellvermehrung zu beobachten [5]. Es ist nicht einmal möglich, dem aktiven Zustand zunächst gut abbauender Kulturen längerfristig zu erhalten. Dieses Phänomen wird "Cooxidation" oder "Cometabolismus" genannt. Diese Betrachtungen machen klar, daß ein solcher Prozeß nicht als "Ein-Stoff-Prozeß" gefahren werden kann. Nur bei kontinuierlich oder zumindest periodisch erfolgender Zufuhr eines gut metabolisierbaren Substrates (Isopren, Methan o.ä.) kommt es zu hohen, stabilen Abbauleistungen. Diese sog. Cosubstrate dienen als Biomasse-aufbauende oder erhaltende Stoffe und sorgen für die Synthese geeigneter Enzyme, die den Abbau der zu cometabolisierenden Substrate bewirken. Die praktische Realisierung solcher Mehrsubstratsysteme stellt ein reizvolles verfahrenstechnisches Problem dar, umsomehr als die abzubauenden Schadstoffe eine Inaktivierung vieler Abbauenzyme bewirken können $[6,7]$.

Hochchlorierte Kohlenwasserstoffe können sich einem aeroben Angriff durch Oxygenaseenzyme auch völlig entziehen. In diesem Fall kann es notwendig werden, die Reinigungsanlage anaerob zu fahren. Hierbei wird der chlorierte Kohlenwasserstoff als Elektronenakzeptor benützt und reduktiv dechloriert. Gegenwärtig häufig bearbeitet werden Chlorethan, 1,2-Dichlorethan und Tetrachlormethan [8,9]. Aufgabe der Grundlagenforschung auf diesem Gebiet ist es, die Abbaugeschwindigkeit dieser anaeroben Vorgänge zu erhöhen und die Prozeßführung zu stabilisieren.

\subsection{Simultaner Abbau von chlor- und methylsubstituierten Aromaten}

Beim Metabolismus von Stoffgemischen kann der Fall eintreten, da $B$ bestimmte Stoffe als Einzelsubstanzen zwar leicht abbaubar sind, im Gemisch verabreicht sich jedoch wie schwerabbaubare Substanzen verhalten $[10,11]$. Dies sei im folgenden am Beispiel eines Gemisches von methyl- und chlorsubstituierten Aromaten eingehend dargestellt. Wie eingangs beschrieben, ist der Abbau von BTX-Aromaten wie 


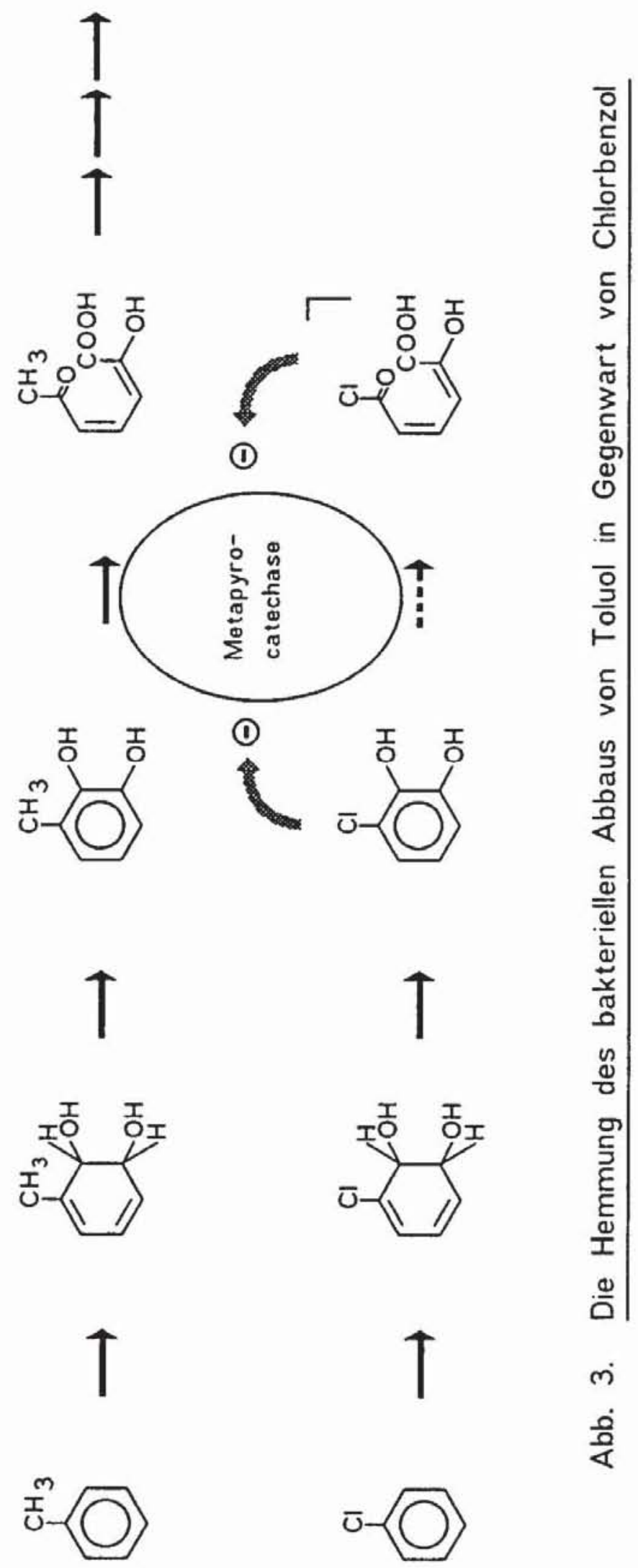


Benzol, Toluol oder Xylol relativ unproblematisch (Abb. 1). Als zentrale Intermediate entstehen in der Regel (alkylsubstituierte) Brenzcatechine, die über Wege der sog. meta-Spaltung weiter abgebaut werden. Konfrontiert man nun Bakterien, die zum BTX-Abbau befähigt sind, mit chlorsubstituierten Aromaten (z. B. Chlorbenzol oder Dichlorbenzol), dann kommt es unter Schwarzfärbung zur Akkumulation von Zwischenprodukten und einem Absterben der Kulturen. In einer technischen Reinigungsanlage wäre damit ein unerwünschter scharfer Leistungsabfall verbunden. Hervorgerufen wird dieses Phänomen durch die "Selbstmord"-Inaktivierung des SchlüsselEnzyms des BTX-Abbaus, der Brenzcatechin-2,3-Dioxygenase durch chlorsubstituierte Analoga (Abb. 3). Diese Inaktivierung führt dazu, daß zunächst weiter angelieferte, sehr toxische Intermediate, sog. Brenzcatechine, nicht weiter abgebaut werden und aufgrund ihrer chemischen Reaktivität zu schwarzgefärbten Polymeren abreagieren. Eine solche Bildung toxischer Intermediate aus relativ untoxischen Ausgangsprodukten läßt sich allerdings auch bei Abbauprozessen für andere Substanzen beobachten. So kann bei mangelhafter ProzeBführung Styrol in Styrolepoxid und Trichlorethylen zu krebserregendem Vinylchlorid umgesetzt werden.

Im Falle des Metabolismus von Chloraromaten durch AlkylaromatenAbbauer kann es auch, je nach Substitutionsart des jeweiligen intermediär entstehenden Brenzcatechin-Derivates, zu einem weitergehenden Metabolismus von chlorsubstituierten Brenzcatechinen über Wege der meta-Spaltung kommen. Immer endet der Abbau jedoch auf einer der folgenden metabolischen Stufen und chlorsubstituierte Endprodukte werden ausgeschieden. Dies bedeutet, daß ein Gemisch von Chlor- und Methylaromaten zwar teilweise abgebaut wird, dabei aber letztlich nur eine Umwandlung bzw. Festlegung der Substanzen in einer festen Matrix erfolgt. Da über die toxikologischen Eigenschaften dieser Umwandlungsprodukte nichts bekannt ist, ist ein solches "Reinigungsverfahren" von äußerst zweifelhaftem Wert.

Alle Ergebnisse der Grundlagenforschung belegen nun eindeutig, da $B$ chlorsubstituierte Aromaten nur über einen anderen Abbauweg als den meta-Weg, nämlich den sog. ortho-Weg dissimiliert werden können (Abb. 4). Dabei werden die Chlorsubstituenten nach Ringspaltung chlorsubstituierter Brenzcatechine auf einer nicht-aromatischen Stufe eliminiert. Allerdings tritt hier das Problem auf, da B alkylsubstituierte Aromaten nun zu zunächst nicht weiter abbaubaren "dead end"Produkten, den methylsubstituierten Lactonen transformiert werden (Abb. 4).

Es gelang jedoch, Bakterien zu selektieren, die solche Lactone umsetzen und als Nahrungsquelle nutzen können [12]. Diese Abbaufähigkeiten wurden mit Sequenzen für den produktiven, d. h. energieliefernden Abbau von chlorsubstituierten Aromaten in einem Bakterium durch Anwendung klassisch-genetischer wie gentechnologischer Verfahren zusammengeführt. Solcherart geschaffene Konstruktionsstämme waren in der Lage, methyl- und chlorsubstituierte Aromaten sowohl als Einzelstoffe wie auch im Gemisch vollständig abzubauen.

\section{Ausblick}

Aus den vorangehenden Beispielen wird klar, daß der biologische Abbau von problematischen Verbindungen eben nicht ein sich in jedem Fall spontan einstellender Proze $B$ ist, sondern -im Vorlauf- intensiver Grundlagenforschung bedarf. Erst nach Klärung der Probleme, die beim biologischen Abbau entstehen, wird ein biologisches Sanierungs- 


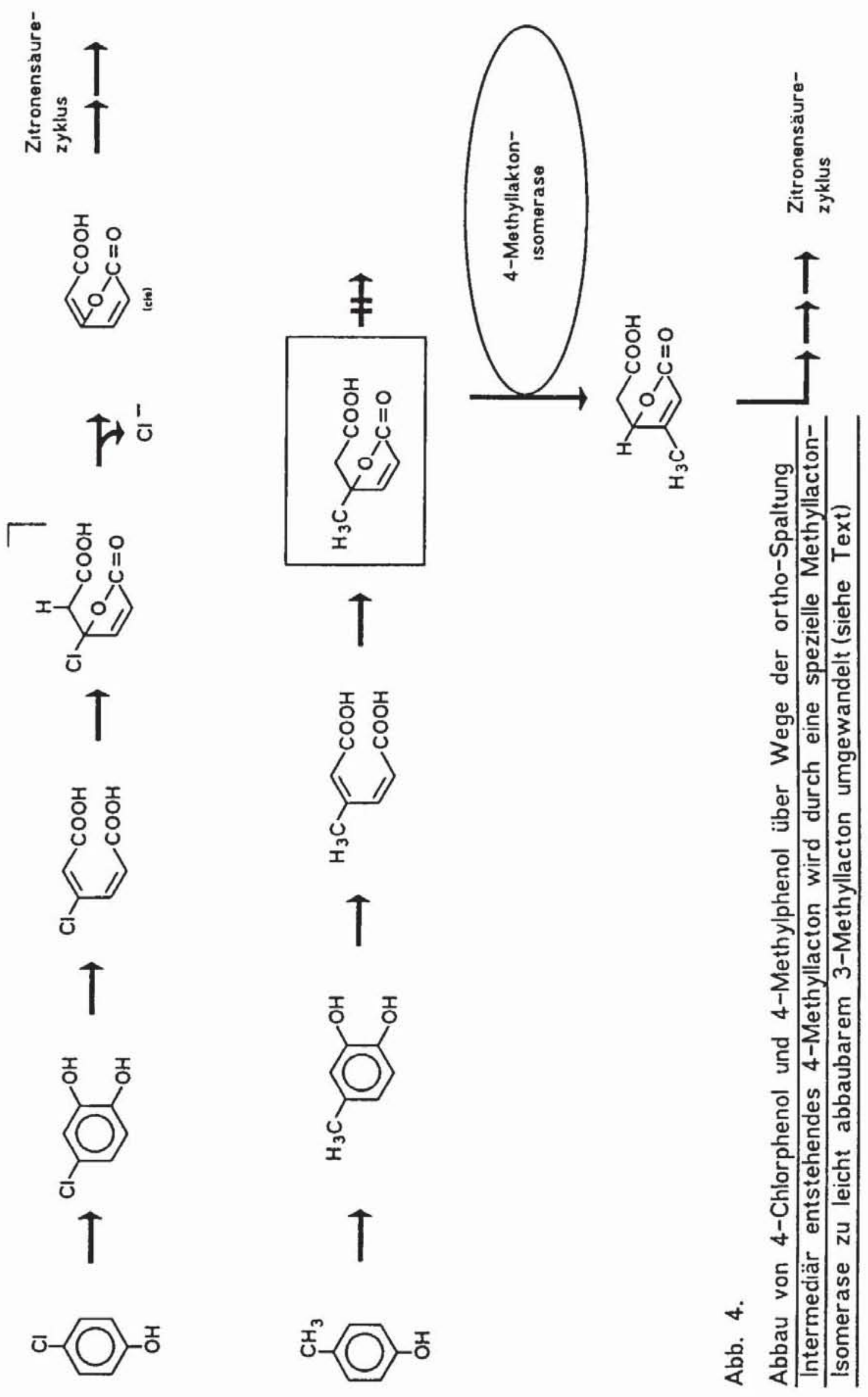


konzept zugänglich. Hier öffnet sich ein umfangreıches Feld, das für weitere Forschungsarbeiten von der Untersuchung der Biochemie spezieller Abbauvorgänge reicht bis zur Lösung physikalischchemischer Probleme, z. B. der limitierenden Geschwindigkeit des Stoffübergangs stark lipophıler Abbaukomponenten in die abbauenden Zellen hinein. Wie beim Beispiel des Abbaus "inkompatibler" Gemische gezeigt, kann es sogar notwendig werden, klassischgenetische oder gar gentechnologische Lösungsansätze ins Auge zu fassen. Obwohl viele grundlegende Forschungsanstrengungen auf diesem komplexen Gebiet schon unternommen wurden, liegt in der Kombination dieser verschiedenen Lösungsansätze die Möglichkeit begründet, auch bisher als biologisch nicht behandelbare Stoffgruppen "Biologie-fähig" zu machen.

\section{Literatur}

1 K. Fischer (Hrsg.), Biologische Abluftreinigung, expert Verlag, Ehningen, 1990.

2 S. Dagley, The Bacteria,Vol. VI Bacterial Diversity, chap. 5, (L.C. Gunsalus ed.), Academic Press Inc., 1978.

3 D.T. Gibson et al., Biochemistry, 12 (1973), 1520-1528.

4 VDI-Richtlinien Nr. 3477, Verein Deutscher Ingenieure. (1989)

5 J. Ewers et al., Arch. Microbiol. 154 (1990) 410-413.

6 J. Ewers et al., Int. Symp. Environm. Biotechnol., (Koninklijke Vlaamse Ingenieursvereniging eds., ISBN-Nr. 90-5204-011-7 (1991) 77 83.

7 L. P. Wackett et al., Appl. Environm. Microbiol. 55 (1989) 2723-2725.

8 C. Egli et al., FEMS Microbiol. Lett. 43 (1987) 257-261.

9 C. Holliger et al., Biodegradation 1 (1990) 253-261.

10 K.-H. Engesser et al., Recent advances in microbial ecology, Proceed. 5th Intern. Symp. Microbial Ecol., (Hattori et al. eds.) (1989) 622-626.

11 H.-J. Knackmuss, Biochem. Soc. Symp. 48(1983) 173-190

12 D. H. Pieper et al., FEMS Lett. 29 (1985).

\section{Danksagung}

Herrn Prof. H.-J. Knackmuss darf ich für viele Anregungen und wertvolle Diskussionen danken. 